The compl ex refractive indices of the I i quid crystal mixture E7 in the ter aher $t$ zf r equencyr ange

\begin{tabular}{|l|l|}
\hline 著者 & $\begin{array}{l}\text { Yang Chan- Shan, Li n Chi a Jen, Pan Ru- Pi n, Que } \\
\text { Chr i st opher T., Yamanto o Kohj i, Tani Masahi ko, } \\
\text { Pan Ci - Li ng }\end{array}$ \\
\hline $\begin{array}{l}\text { j our nal or } \\
\text { publ i cat i on ti t l e }\end{array}$ & Journal of the Opt i cal Soci et y of Aner i ca B \\
\hline vol une & 27 \\
\hline number & 9 \\
\hline page r ange & $1866-1873$ \\
\hline year & $2010-09$ \\
\hline URL & ht t p: //hdl . handl e. net /10098/3028 \\
\hline
\end{tabular}




\title{
The complex refractive indices of the liquid crystal mixture E7 in the terahertz frequency range
}

\author{
Chan-Shan Yang, ${ }^{1}$ Chia-Jen Lin, ${ }^{2}$ Ru-Pin Pan, ${ }^{2,4}$ Christopher T. Que, ${ }^{3}$ Kohji Yamamoto, ${ }^{3}$ Masahiko Tani, ${ }^{3}$ and \\ Ci-Ling Pan ${ }^{1,5}$ \\ ${ }^{1}$ Department of Physics, National Tsing Hua University, No. 101, Section 2, Kuang Fu Road, Hsinchu, \\ Taiwan 30013, China \\ ${ }^{2}$ Department of Electrophysics, National Chiao Tung University, 1001 Ta Hsueh Road, Hsinchu, \\ Taiwan 30010, China \\ ${ }^{3}$ Research Center for Development of Far-Infrared Region, University of Fukui, 3-9-1 Bunkyo, Fukui 910-8507, Japan \\ ${ }^{4}$ e-mail: Rpchao@mail.nctu.edu.tw \\ ${ }^{5}$ e-mail: clpan@phys.nthu.edu.tw
}

Received June 18, 2010; revised July 1, 2010; accepted July 5, 2010;

posted July 8, 2010 (Doc. ID 130294); published August 23, 2010

\begin{abstract}
We have used terahertz time-domain spectroscopy to investigate the complex optical constants and birefringence of a widely used liquid crystal mixture $\mathrm{E} 7 \mathrm{in}$ both nematic and isotropic phases $\left(26^{\circ} \mathrm{C}-70^{\circ} \mathrm{C}\right)$. The extinction coefficient of E7 at room temperature is less than 0.035 and without sharp absorption features in the frequency range of $0.2-2.0 \mathrm{THz}$. The extraordinary $\left(n_{e}\right)$ and ordinary $\left(n_{o}\right)$ indices of refraction at $26^{\circ} \mathrm{C}$ are $1.690-1.704$ and $1.557-1.581$, respectively, giving rise to a birefringence of $0.130-0.148$ in this frequency range. The temperature-dependent $\left(26^{\circ} \mathrm{C}-70^{\circ} \mathrm{C}\right)$ order parameter extracted from the birefringence data agrees with that in the visible region quite well. Further, the temperature gradients of the terahertz optical constants of E7 are also determined. The optical constants of E7 in the terahertz or sub-millimeter wave range are found to deviate significantly from values predicated by the usual extended Cauchy equations used in the visible and near-infrared. () 2010 Optical Society of America
\end{abstract}

OCIS codes: $000.6850,040.2235,120.4530,140.7090,160.1190,160.3710$.

\section{INTRODUCTION}

Due to their relative large birefringence $\left(\Delta n=n_{e}-n_{o}\right)$ from the visible to microwave band, liquid crystals (LCs) have found a variety of applications ranging from display, tunable optical elements, communication and signal processing, beam steering, etc. [1,2]. In order for LCs to be employed at a particular wavelength, it is thus essential that the magnitudes of the extraordinary and ordinary refractive indices, $n_{e}$ and $n_{o}$, as well as the birefringence of the LC are known. The temperature effects on the optical constants are also of interest.

The LC mixture E7, composed of 4-cyano-4' - $n$-pentyl-biphenyl (5CB), 4-cyano-4'-nheptylbiphenyl (7CB), 4-cyano-4'-n-octyloxy-biphenyl, and 4-cyano-4"- $n$-pentyl- $p$-terphenyl, has been widely used in LC devices due to its large birefringence $(\sim 0.2$ in the visible) and wide nematic temperature range $\left(-10^{\circ} \mathrm{C}\right.$ to $59^{\circ} \mathrm{C}$ ). The optical constants of $\mathrm{E} 7$ have been studied extensively in the past. In the visible (from 656 to $450 \mathrm{~nm}$ ) and at room temperature $\left(25^{\circ} \mathrm{C}\right)$, for example, Li et al. [3] reported that $n_{e}$ of $\mathrm{E} 7$ increases from 1.73 to 1.80 , while its $n_{o}$ also increases slowly from 1.52 to $1.54(\Delta n$ $\sim 0.21-0.26$ ). In the near-infrared region, the same group reported that $\Delta n=0.186$ at $1.55 \mu \mathrm{m} \mathrm{[4]} \mathrm{and} \sim 0.191$ in the wavelength range from 1.611 to $2.833 \mu \mathrm{m}$. The birefringence of $\mathrm{E} 7$ in the mid-infrared region $(2-16 \mu \mathrm{m})$ can be approximated at a constant, $\Delta n \sim 0.18$, outside the vicinity of vibrational bands [5]. At the $\mathrm{CO}_{2}$ laser wavelengths
( $\lambda=10.6 \mu \mathrm{m})$, Wu et al. [4] and Brugioni and Meucci [6] independently determined that $n_{e}=1.69$ and $n_{o}=1.49$ $(\Delta n=0.20)$. In general, the refractive indices of $\mathrm{E} 7$ can be modeled by the extended Cauchy equation from the visible to the infrared [4]. The corresponding birefringence exhibit a general decreasing trend as the wavelength increased over this spectral range. For wavelengths much longer than the mean resonance wavelength $\left(\lambda^{*}\right.$ $\sim 250 \mathrm{~nm}$ ), i.e., infrared and beyond, $\Delta n$ is expected to approach a limiting but appreciable value [5].

In the past decade, terahertz studies ranging from investigations of ultrafast dynamics in materials to medical and environmental sensing and imaging have been actively explored [7-10]. For these and future applications in terahertz communication and surveillance, quasioptical components [11] such as phase modulators [12,13] are increasingly in demand. Liquid-crystal-based terahertz devices are attractive in quasi-optical systems provided LCs exhibit reasonably large birefringence and acceptable transmittance can be found. Indeed, several kinds of LC devices, e.g., phase shifters [14], birefringent [15,16], plasmonic [17], and Fabry-Perot filters [18], polarizers [19], phase gratings [20], and Bragg switches [21], have been demonstrated. The LC mixture E7 (Merck), with a wide nematic temperature range $\left(-10^{\circ} \mathrm{C}\right.$ to $59^{\circ} \mathrm{C}$ ), was the LC of choice in most of these works. At room temperature, we showed that there is no sharp resonance for the optical constants of $\mathrm{E} 7$ in the $0.2-1.2 \mathrm{THz}$ 
range, in which the extraordinary index of refraction, $n_{e}$, varies from 1.69 to 1.80 , while the ordinary index of refraction, $n_{o}$, varies from 1.51 to 1.63 . The terahertz birefringence of E7 is thus $0.12-0.21$. The corresponding imaginary indices of E7 are relatively small $(<0.04)$ [22]. Independently, Jewell et al. [18] determined that $\Delta n$ $=0.15( \pm 0.01)$ for E7 over the same frequency range. In this work, we use terahertz time-domain spectroscopy (THz-TDS) to conduct a detailed study of the frequency dependence $(0.2-2.0 \mathrm{THz})$ and temperature dependence of the complex terahertz optical constants of $\mathrm{E} 7$ in both nematic and isotropic phases $\left(26^{\circ} \mathrm{C}-70^{\circ} \mathrm{C}\right)$. The temperature-dependent terahertz birefringence $\Delta n$ and the order parameter $S$ of $\mathrm{E} 7$ in this spectral range have also been investigated. Further, the temperature gradients of the terahertz optical constants of E7 are also determined. It is shown that the optical constants of E7 in the terahertz or sub-millimeter wave range deviate significantly from values predicated by the usual extended Cauchy equations used in the visible and near-infrared.

\section{EXPERIMENTAL METHODS}

A homogeneously aligned LC cell and a reference cell, schematically shown in Fig. 1, were prepared in this work. The reference cell was constructed by two opticalgrade fused silica windows with thicknesses $d_{1}$ and $d_{2}$. The LC cell was constructed by sandwiching the LC (E7, Merck) between two windows of the aforementioned types with thicknesses $d_{3}$ and $d_{4}$. The thickness of the LC layer was controlled with a Mylar spacer and measured by subtracting the substrate thicknesses from the total cell thickness. The LC layer thickness in this work was $d_{\mathrm{LC}}$ $=0.552 \pm 0.002 \mathrm{~mm}$. We achieved homogeneous alignment of the nematic LC by rubbing the polyimide films on the substrates. The temperature of the LC cell can be varied with an accuracy of $\pm 0.1^{\circ} \mathrm{C}$. The thickness of each of the windows employed was found to be $\sim 3 \mathrm{~mm}$ as measured with a micrometer.

To determine the optical constants, we employed a photoconductive-antenna-based THz-TDS system described previously [23]. Data were taken both in Taiwan and Japan; the results are consistent and complementary. For the frequency range below $0.4 \mathrm{THz}$ and above 1.4 $\mathrm{THz}$, data taken, respectively, in Taiwan and Japan were used in view of the signal to noise performance of the two systems. Between 0.4 and $1.4 \mathrm{THz}$, the data from the two laboratories were identical within experimental error. The THz-TDS in Taiwan was purged with dry nitrogen so
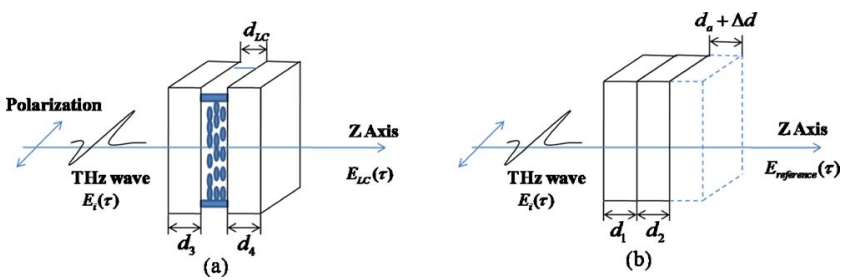

Fig. 1. (Color online) Simplified sketches of the experimental configuration for transmission of ordinary ray through (a) the LC and (b) reference cells. The symbols are defined in the text. The blue ellipsoids in (a) represent schematically the LC molecules, which are homogeneously aligned in the cell. that it could be maintained at a relative humidity of $(4.0 \pm 0.5) \%$. In the experiments, the reference of the LC cell was placed in the THz-TDS at the position where the terahertz beam was collimated. For measurements of the extraordinary and ordinary indices of refraction, $n_{e}$ and $n_{o}$, we rotated the LC cell such that its director was either perpendicular or parallel to the polarization direction of the incident terahertz wave.

\section{EXTRACTION AND ANALYSIS OF THE OPTICAL CONSTANTS}

For a monochromatic plane terahertz wave propagating through the cells at normal incidence (see Fig. 1), we can write the electric field of the terahertz wave transmitted through the reference cell as

$$
\begin{aligned}
E_{\mathrm{ref}}(\omega)= & E_{0}(\omega) \tilde{t}_{a w} \tilde{t}_{w a} \exp \left[-i(\omega / c) \tilde{n}_{w}\left(d_{1}+d_{2}\right)\right] \\
& \times \exp \left[-i(\omega / c) \tilde{n}_{a}\left(d_{a}+\Delta d\right)\right]
\end{aligned}
$$

where $E_{0}(\omega)$ is the electric field of the incident terahertz wave. The ratios $\widetilde{t}_{a w}=2 \widetilde{n}_{a} /\left(\widetilde{n}_{a}+\widetilde{n}_{w}\right)$ and $\widetilde{t}_{w a}=2 \widetilde{n}_{w} /\left(\widetilde{n}_{w}\right.$ $\left.+\tilde{n}_{a}\right)$ are the transmission coefficients of the terahertz signal from air to the fused silica substrate and from the substrate to air, respectively; $\tilde{n}_{w}$ and $\tilde{n}_{a}$ are the refractive indices of the substrate and air. Here, the slight difference in thicknesses, $d_{1}+d_{2}$ and $d_{3}+d_{4}$, of the reference and LC cells is taken into account by the term $\Delta d=\left(d_{3}+d_{4}\right)-\left(d_{1}\right.$ $\left.+d_{2}\right) ; d_{a}$ is thickness of a mass of air assumed to have the same value as $d_{\mathrm{LC}}$. Similarly, the electric field of the terahertz wave passed through the LC cell can be written as

$$
\begin{aligned}
E_{\mathrm{LC}}(\omega)= & E_{0}(\omega) \widetilde{t}_{a w} \widetilde{t}_{w-\mathrm{LC}} \widetilde{t}_{\mathrm{LC}-w} \widetilde{t}_{w a} \\
& \times \exp \left[-i(\omega / c) \widetilde{n}_{w}\left(d_{3}+d_{4}\right)\right] \widetilde{F P}_{\mathrm{LC}}\left(\left(\omega, d_{\mathrm{LC}}\right),\right.
\end{aligned}
$$

where $\tilde{t}_{w-\mathrm{LC}}=2 \tilde{n}_{w} /\left(\tilde{n}_{w}+\tilde{n}_{\mathrm{LC}}\right)$ and $\tilde{t}_{\mathrm{LC}-w}=2 \tilde{n}_{\mathrm{LC}} /\left(\tilde{n}_{\mathrm{LC}}+\tilde{n}_{w}\right)$ are the transmission coefficients from fused silica substrate to the LC layer and from the LC layer to the substrate interfaces, respectively. The $\widetilde{F P}_{\mathrm{LC}}$ parameter is the Fabry-Perot coefficient for multiple reflections of the terahertz beam in the nematic LC cell, and can be written as

$$
\widetilde{F P}_{\mathrm{LC}}\left(\omega, d_{\mathrm{LC}}\right)=\sum_{m=0}^{N}\left(\widetilde{r}_{\mathrm{LC}-w}^{2 m} \exp \left\{-i(\omega / c)\left[\widetilde{n}_{\mathrm{LC}} d_{\mathrm{LC}}(2 m+1)\right]\right\}\right)
$$

with a thickness of $d_{\mathrm{LC}}$, and $\tilde{n}_{\mathrm{LC}}$ is the complex refractive index of the LC. In expressing the transmitted terahertz wave as in Eqs. (1) and (2), we assumed that the multiple reflections in the substrates can be ignored. Experimentally, this is done by choosing the measurement time window such that the multiple reflections are excluded.

Using Eqs. (1) and (2), we can write the transmission coefficient of the LC cell, normalized to that of the reference, as 


$$
\begin{aligned}
T(\omega)= & E_{\mathrm{LC}}(\omega) / E_{\mathrm{ref}}(\omega)=\widetilde{t}_{w-\mathrm{LC}} \widetilde{t}_{\mathrm{LC}-w} \exp \left\{-i(\omega / c)\left[\Delta d\left(\tilde{n}_{w}-\tilde{n}_{a}\right)\right.\right. \\
& \left.\left.+d_{\mathrm{LC}}\left(\widetilde{n}_{\mathrm{LC}}-\tilde{n}_{a}\right)\right]\right\} \sum_{m=0}^{N} \tilde{r}_{\mathrm{LC}-w}^{2 m} \exp \left[-i 2 m(\omega / c) \tilde{n}_{\mathrm{LC}} d_{\mathrm{LC}}\right] .
\end{aligned}
$$

Expressing $\tilde{n}_{\mathrm{LC}}, \tilde{n}_{w}$, and $\tilde{n}_{a}$ into their real and imaginary parts, $\tilde{n}_{\mathrm{LC}}=n_{\mathrm{LC}}+i \kappa_{\mathrm{LC}}, \tilde{n}_{w}=n_{w}+i \kappa_{w}$, and $\tilde{n}_{a}=n_{0}$, we can write the complex refractive index of the LC as follows:

$$
\begin{aligned}
n_{\mathrm{LC}}= & n_{0}+\left[\arg (T(\omega))-\arg \left(\tilde{t}_{w-\mathrm{LC}} \widetilde{t}_{\mathrm{LC}-w}\right.\right. \\
& \times \exp \left[-\omega\left(\Delta d \kappa_{w}+d_{\mathrm{LC}} \kappa_{\mathrm{LC}}\right) / c\right] \sum_{m=0}^{N} \tilde{r}_{\mathrm{LC}-w}^{2 m} \\
& \left.\left.\times \exp \left(-i 2 m \tilde{n}_{\mathrm{LC}} d_{\mathrm{LC}} \omega / c\right)\right)\right] c /\left(\omega d_{\mathrm{LC}}\right) \\
& -\Delta d\left(n_{w}-n_{0}\right) / d_{\mathrm{LC}}, \\
\kappa_{\mathrm{LC}}= & \kappa_{w} \Delta d / d_{\mathrm{LC}}-\ln \left[|T(\omega)| /\left\{\sum_{m=0}^{N} \tilde{r}_{\mathrm{LC}-w}^{2 m}\left|\widetilde{t}_{w-\mathrm{LC}} \widetilde{t}_{\mathrm{LC}-w}\right|\right\}\right] c /\left(\omega d_{\mathrm{LC}}\right) . \\
& \times \exp \left[-i 2 m(\omega / c) \tilde{n}_{\mathrm{LC}} d_{\mathrm{LC}}\right] \mid
\end{aligned}
$$

Previous studies $[14,18]$ have shown that there is no resonance in the spectral range $(0.2-1.2 \mathrm{THz})$ for either $n_{e}$ or $n_{o}$ E7. It is then expected that its ordinary and extraordinary refractive indices can be fitted by the threeparameter Cauchy formula [4]

$$
n_{o, e}=A_{o, e}+B_{o, e} \lambda^{-2}+C_{o, e} \lambda^{-4} .
$$

For several LCs, including E7 at room temperature, Li et al. [4] determined the fitting parameters. The birefrin-

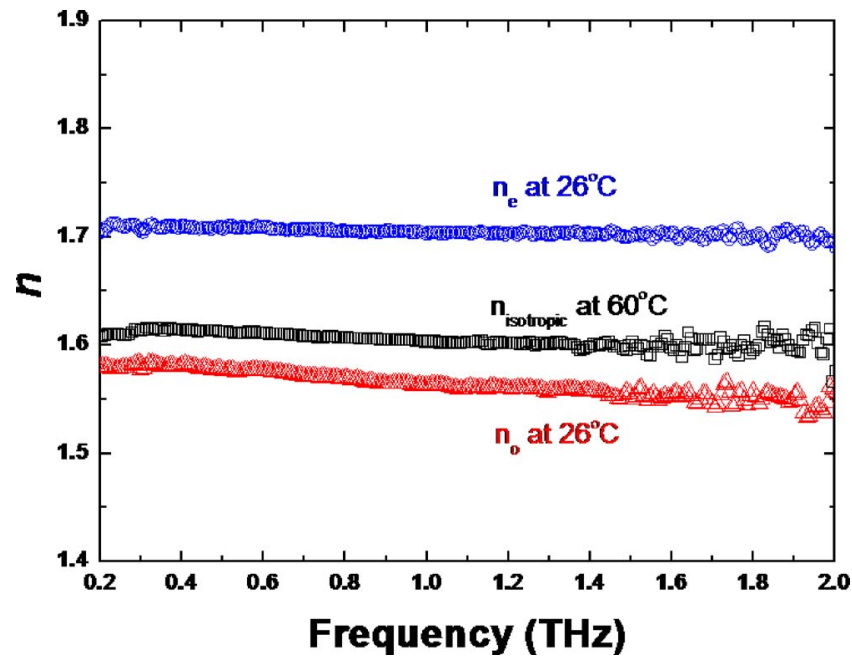

Fig. 2. (Color online) The real refractive indices of E7 are plotted as functions of frequency. The blue circles and the red triangles are the extraordinary and ordinary refractive indices of $\mathrm{E} 7, n_{e}$ and $n_{o}$, at $26^{\circ} \mathrm{C}$, respectively. The black squares are the real refractive indices of $\mathrm{E} 7$ in the isotropic phase $\left(\right.$ at $\left.60^{\circ} \mathrm{C}\right)$. The error bars are comparable to or smaller than the symbols.

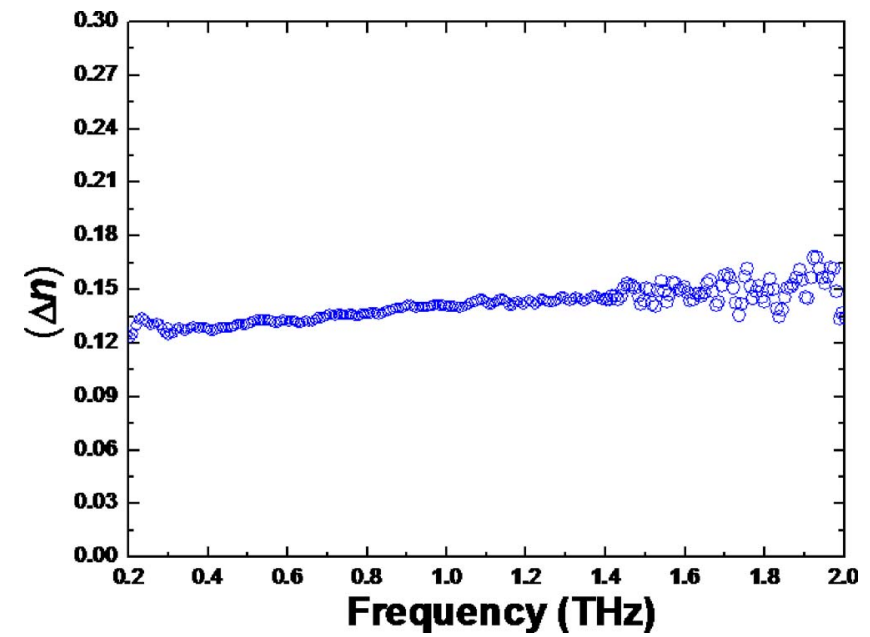

Fig. 3. (Color online) Frequency dependence of the birefringence of $\mathrm{E} 7$ measured at $26^{\circ} \mathrm{C}$. The error bars are comparable to or smaller than the symbols.

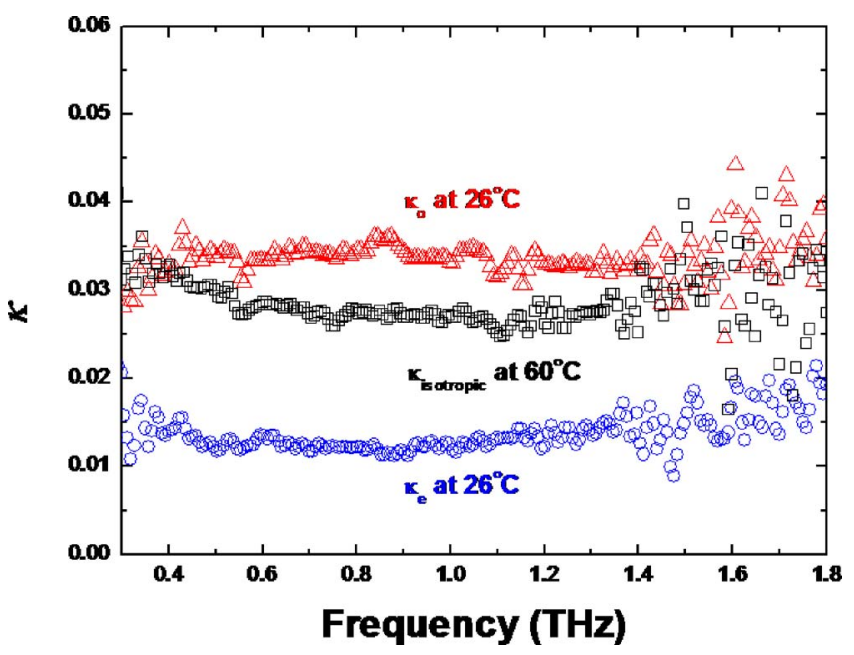

Fig. 4. (Color online) The imaginary refractive indices of E7 are plotted as functions of frequency. The blue circles and the red triangles are $\kappa_{e}$ and $\kappa_{o}$ at $26^{\circ} \mathrm{C}$, respectively. The black squares are the imaginary refractive indices of $\mathrm{E} 7$ in the isotropic phase (at $60^{\circ} \mathrm{C}$ ). The error bars are comparable to or smaller than the symbols.

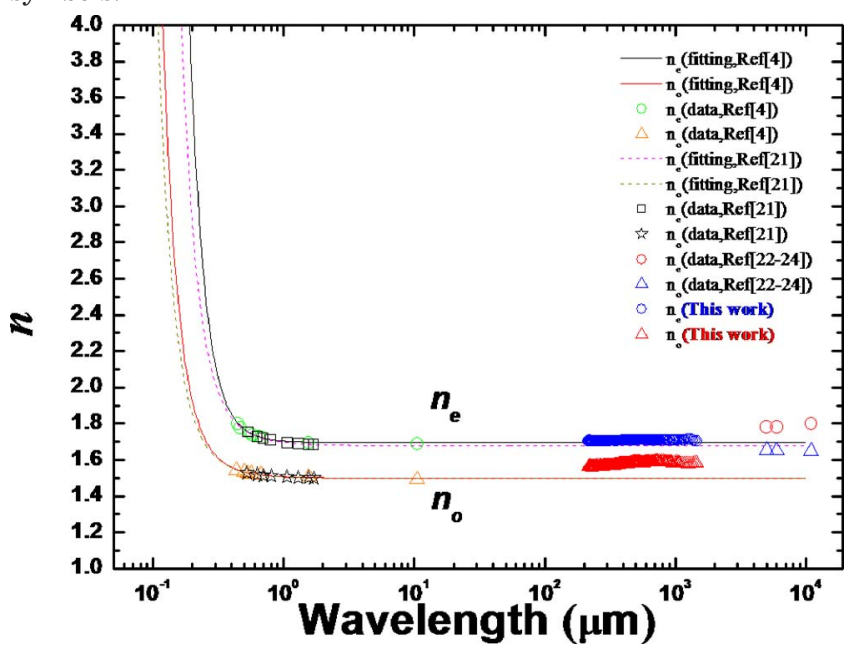

Fig. 5. (Color online) The refractive indices of E7 reported in the literature from the near-infrared [4,27], mid-infrared [4], to the millimeter [28-30] waves together with those of this work in the millimeter and sub-millimeter wave ranges. 


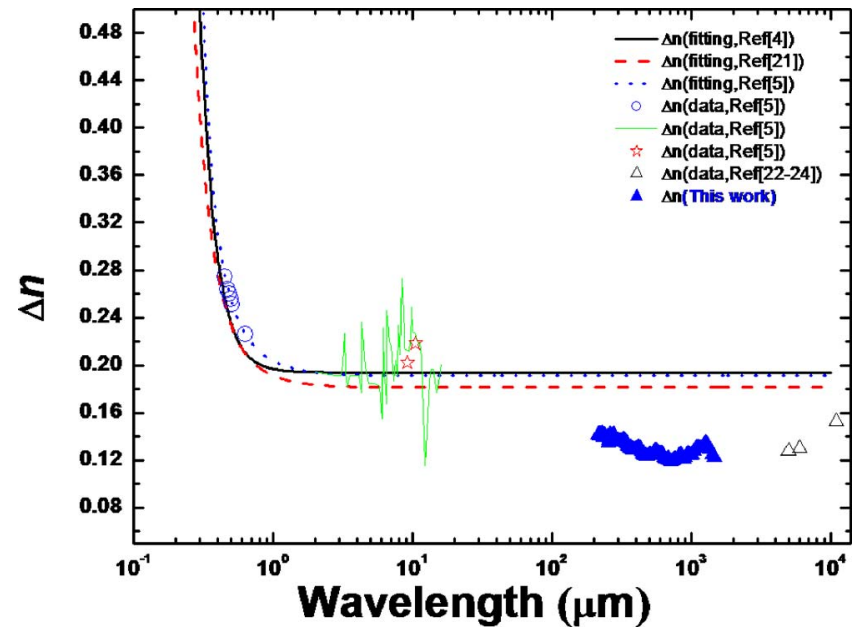

Fig. 6. (Color online) The birefringence of E7 reported in the literature from the visible [5], near-infrared [4,27], mid-infrared [4], to the millimeter [28-30] waves together with those of this work in the millimeter and sub-millimeter wave ranges.

gence of the LC can then be calculated using Eq. (7).

Next, we consider the temperature effect on the optical constants of the LC. Confirming the results of Li et al. [24] in the visible, we also observed a linear temperature dependence of the average refractive indices of $\mathrm{E} 7$ in the farinfrared (see discussions in Subsection 4.B), $\langle n\rangle=\left(n_{e}\right.$ $\left.+2 n_{o}\right) / 3$, on temperature, i.e.,

$$
\langle n(T)\rangle=A-B T
$$
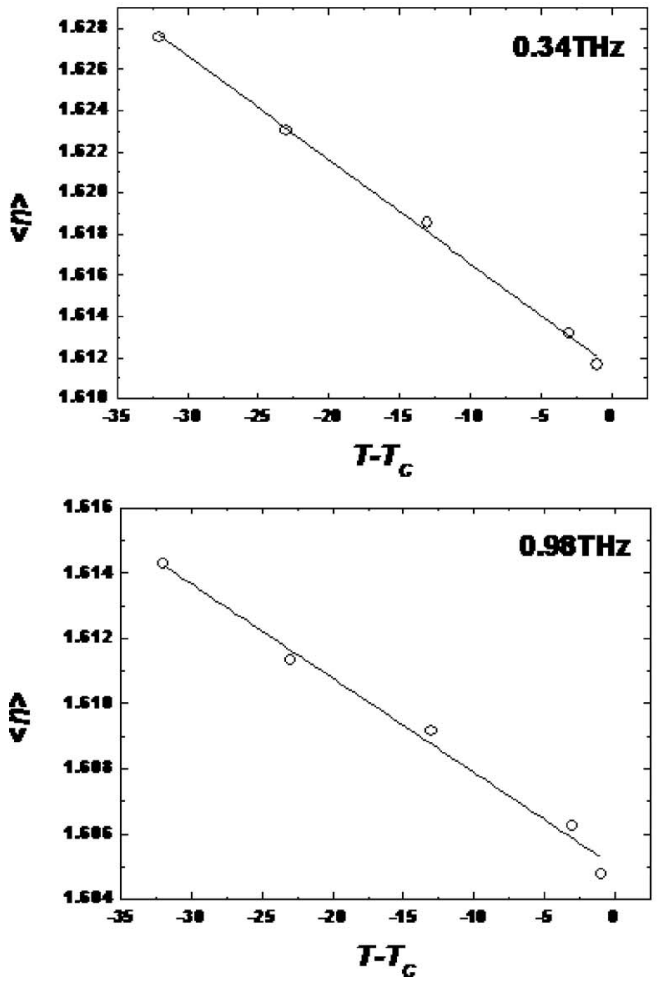

Furthermore, because $\Delta n$ is linearly proportional to $S$, its temperature dependence can be written as

$$
\Delta n(T)=(\Delta n)_{0} S=(\Delta n)_{0}\left(1-T / T_{c}\right)^{\beta},
$$

where $(\Delta n)_{0}$ is the birefringence of the $\mathrm{LC}$ at $T=0 \mathrm{~K}, \beta$ is a material constant which is not too sensitive to the molecular structure of $\mathrm{LCs}$, and $T_{c}$ is the clearing point of the LC [24].

With Eqs. (8) and (9), we arrive at the four-parameter model for the temperature dependence of $n_{e}$ and $n_{o}$,

$$
\begin{aligned}
& n_{e}(T)=A-B T+\left(2(\Delta n)_{0} / 3\right)\left(1-T / T_{c}\right)^{\beta}, \\
& n_{o}(T)=A-B T-\left((\Delta n)_{0} / 3\right)\left(1-T / T_{c}\right)^{\beta} .
\end{aligned}
$$

In the above two equations, parameters $A$ and $B$ can be obtained by fitting the temperature-dependent $\langle n\rangle$ for a given wavelength, while $(\Delta n)_{0}$ and $\beta$ can be determined by fitting $\Delta n(T)$.

Furthermore, we can derive the temperature gradients of $n_{e}$ and $n_{o}$ from Eqs. (10) and (11) as follows [25]:

$$
\begin{gathered}
\mathrm{d} n_{e} / \mathrm{d} T=-B-2 \beta(\Delta n)_{0} /\left[3 T_{c}\left(1-T / T_{c}\right)^{1-\beta}\right], \\
\mathrm{d} n_{o} / \mathrm{d} T=-B+\beta(\Delta n)_{0} /\left[3 T_{c}\left(1-T / T_{c}\right)^{1-\beta}\right] .
\end{gathered}
$$

These quantities are of interest in the design of LC devices based on laser-induced thermal effects in LCs [26]. The temperature gradient of the birefringence could also be significant for many applications based on $\Delta n$.
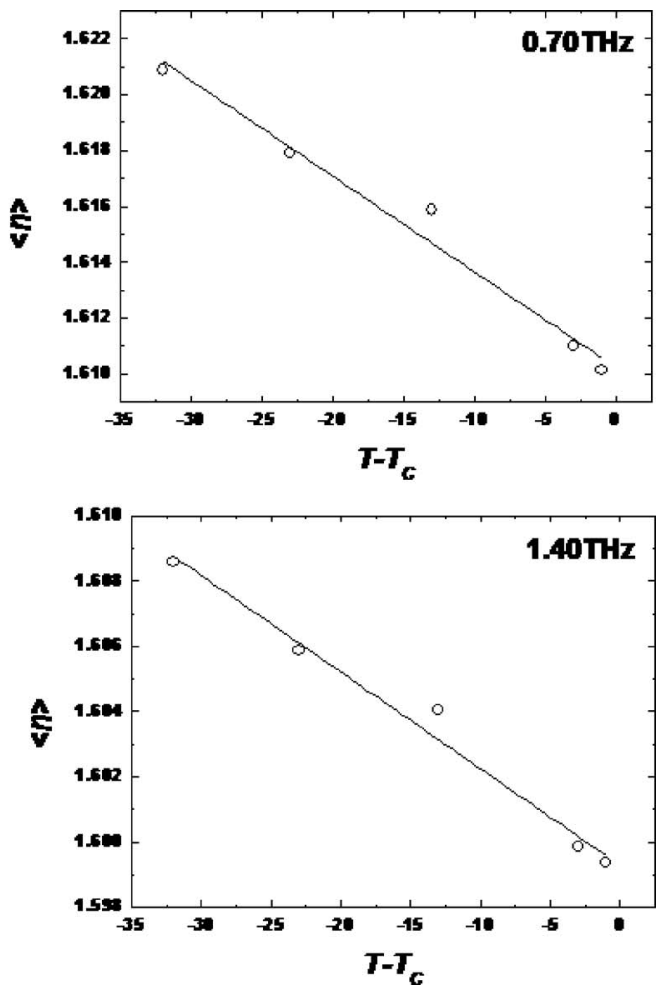

Fig. 7. Average refractive indices of E7 versus $T-T_{c}$ at frequencies of $0.34,0.70,0.98$, and $1.40 \mathrm{THz}$. The open circles represent the experimental data and the solid lines are the results of fitting. 
Table 1. The Fitting Parameters of Eq. (8) for Frequencies from 0.34 to $1.40 \mathrm{THz}$

\begin{tabular}{ccc}
\hline $\begin{array}{c}\text { Frequency } \\
(\mathrm{THz})\end{array}$ & $A$ & $\begin{array}{c}B \\
\left(\mathrm{~K}^{-1}\right)\end{array}$ \\
\hline 0.34 & 1.7787 & $5.05 \times 10^{-4}$ \\
0.41 & 1.7804 & $5.03 \times 10^{-4}$ \\
0.53 & 1.7505 & $4.15 \times 10^{-4}$ \\
0.70 & 1.7235 & $3.42 \times 10^{-4}$ \\
0.80 & 1.7165 & $3.24 \times 10^{-4}$ \\
0.89 & 1.7010 & $2.90 \times 10^{-4}$ \\
0.98 & 1.7006 & $2.89 \times 10^{-4}$ \\
1.10 & 1.6945 & $2.78 \times 10^{-4}$ \\
1.19 & 1.6743 & $2.16 \times 10^{-4}$ \\
1.29 & 1.6674 & $1.90 \times 10^{-4}$ \\
1.40 & 1.6974 & $2.96 \times 10^{-4}$ \\
\hline
\end{tabular}

\section{RESULTS AND DISCUSSIONS}

\section{A. Complex Optical Constants}

For the range of $0.2-2.0 \mathrm{THz}$, the extraordinary $\left(n_{e}\right)$ and ordinary $\left(n_{o}\right)$ indices of refraction of $\mathrm{E} 7$ at $26^{\circ} \mathrm{C}$ and its refractive index in the isotropic phase $\left(60^{\circ} \mathrm{C}\right)$ are shown in Fig. 2. Clear anisotropy in nematic phase was observed with $n_{e}=1.690-1.704$ and $n_{o}=1.557-1.581$. The refractive indices decrease slightly with increasing frequency from 0.2 to $2 \mathrm{THz}$. This general trend is also observed in recent study of the terahertz birefringence of the homologous series of nematic cyanobiphenyls by Lin et al. [20]. The corresponding terahertz birefringence $(\Delta n)$ of $\mathrm{E} 7$ at $26^{\circ} \mathrm{C}$ is about $0.130-0.148$ and plotted as a function of
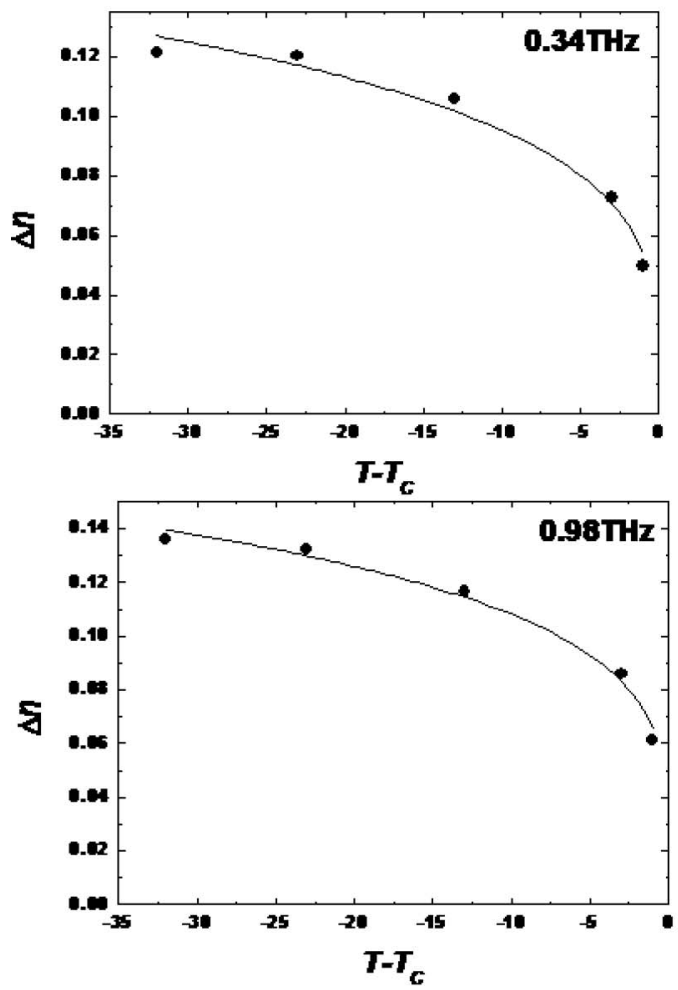

frequency in Fig. 3. This is comparable to the reported birefringence of $\mathrm{E} 7$ in the microwave range $(\Delta n$ $=0.13-0.15)$, but somewhat smaller than those in the visible band $(\Delta n=0.21-0.26)$. The extinction coefficient of E7 is shown in Fig. 4 with $\kappa_{e}=0.010-0.020$ and $\kappa_{o}$ $=0.028-0.042$. In other words, the absorption coefficients for ordinary and extraordinary waves at $1 \mathrm{THz}$ are $\alpha_{e}=5.001 \mathrm{~cm}^{-1}$ and $\alpha_{o}=15.573 \mathrm{~cm}^{-1}$, respectively. Data shown in Figs. 2 and 4 indicate that there is no sharp absorption feature for E7 in this range. Figure 4 also shows that $\mathrm{E} 7$ is dichroic with a dichroic ratio, $R=\alpha_{o} / \alpha_{e}$, being 3.11 at $1 \mathrm{THz}$.

For comparison, we have organized the refractive indices of E7 reported in the literature from the visible [5], near-infrared [4,27], mid-infrared [4], to the millimeter waves [28-30] together with those of ours in the millimeter and sub-millimeter wave ranges. This is shown in Fig. 5. The data from the visible to the mid-infrared can be fitted quite well by Eq. (7). The refractive indices in the terahertz (sub-millimeter wave), millimeter wave, and microwave ranges, however, deviate significantly from the fitting curves. Similarly, the available birefringence data across the entire spectrum are shown in Fig. 6. The fitting curves were calculated using Eq. (7). Clearly the terahertz birefringence of E7 is smaller than that predicted from the visible birefringence.

The above observations could be qualitatively explained. It is well-known that dispersion could vary significantly near resonances. Cyanobiphenyls, such as 5CB and $7 \mathrm{CB}$, are major components of E7. $5 \mathrm{CB}$ is known to have broad absorption features near 100, 140, and $165 \mathrm{~cm}^{-1}$, i.e., $\sim 3-6 \mathrm{THz}$ to [31]. In particular, there is a
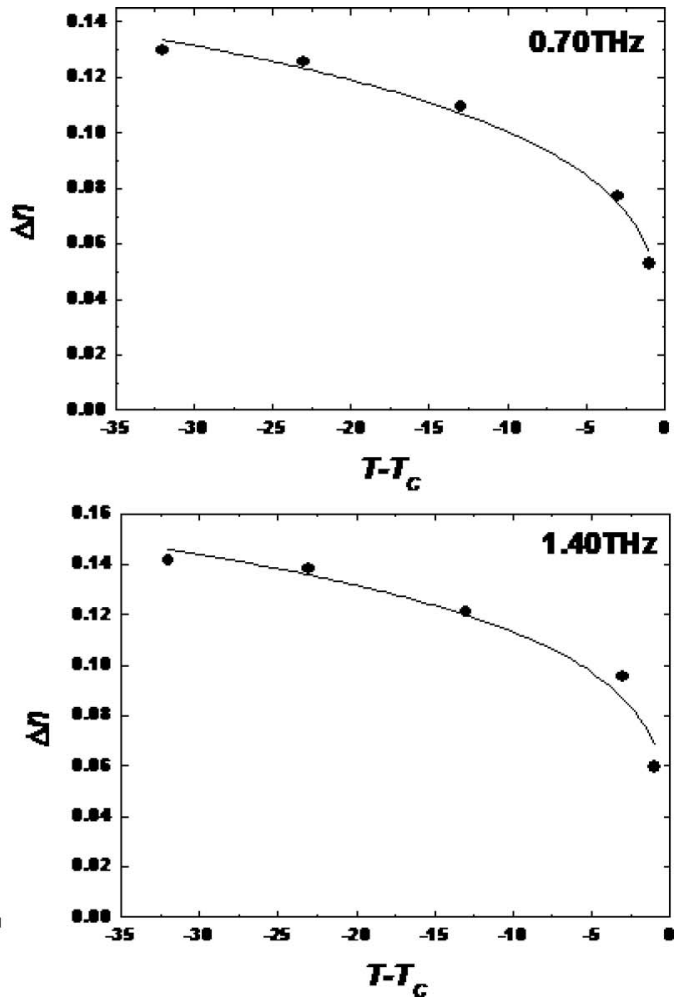

Fig. 8. Birefringence of $\mathrm{E} 7$ versus $T-T_{c}$ at frequencies of $0.34,0.70,0.98$, and $1.40 \mathrm{THz}$. The closed circles represent the experimental data and the solid curves are the results of fitting. 
Table 2. The Fitting Parameters of Eq. (9) for Frequencies from 0.34 to $1.40 \mathrm{THz}$

\begin{tabular}{ccc}
\hline $\begin{array}{c}\text { Frequency } \\
(\mathrm{THz})\end{array}$ & $(\Delta n)_{0}$ & $\beta$ \\
\hline 0.34 & 0.2257 & 0.2466 \\
0.41 & 0.2190 & 0.2400 \\
0.53 & 0.2356 & 0.2495 \\
0.70 & 0.2376 & 0.2461 \\
0.80 & 0.2312 & 0.2342 \\
0.89 & 0.2397 & 0.2306 \\
0.98 & 0.2333 & 0.2196 \\
1.10 & 0.2374 & 0.2098 \\
1.19 & 0.2401 & 0.2163 \\
1.29 & 0.2424 & 0.2157 \\
1.40 & 0.2439 & 0.2195 \\
\hline
\end{tabular}

broad shoulder extending from the sub-terahertz frequencies to the absorption band near $\sim 100 \mathrm{~cm}^{-1}$ assigned to the libration of the rigid molecule around its long axis. The far-infrared absorption spectrum of 7CB exhibits similar profiles with absorption bands near $\sim 100,160$, and $180 \mathrm{~cm}^{-1}$ [32]. Thus we can qualitatively understand the deviation of the terahertz refractive indices of $\mathrm{E} 7$ from Eq. (7), the three-parameter Cauchy equation, as it does not take into account these far-infrared resonances.

\section{B. Temperature Effect}

Following Li et al. [24], we used the four-parameter model $\left[A, B,(\Delta n)_{0}, \beta\right]$ to investigate the effect of temperature on the refractive index of E7. The measured data on the terahertz refractive indices $\left(n_{e}, n_{o}\right)$ allow the calculation of the average refractive indices $(\langle n\rangle)$ and birefringence $(\Delta n)$. Equation (8) is then used to fit the average refractive indices and get the parameters $A$ and $B$. Results are shown in Fig. 7. Here, the open circles represent the experimental data and the solid lines are the results of fitting. We also list the fitting parameters in Table 1 . It can be seen that $\langle n\rangle$ decreases as the temperature increases. The value of $B$ is around $10^{-4} / \mathrm{K}$, consistent with the result of Li et al. [4].

We have also plotted $\Delta n$ as a function of $T$ - $T_{c}$, where $T_{c}$ is the clearing point (see Fig. 8). Equation (9) is then used to fit the birefringence and obtain the parameters $(\Delta n)_{0}$ and $\beta$, which are summarized in Table 2. The temperature-dependent order parameter extracted from $\Delta n\left(S=0.58\right.$ at $1 \mathrm{THz}$ and $\left.26^{\circ} \mathrm{C}\right)$ agrees with values measured in the visible and near-infrared regions quite well. This is another indication that the data in the terahertz range are trustworthy.

Lastly, we substitute these two sets of parameters summarized in Tables 1 and 2 into Eqs. (10) and (11) and obtain the fitted refractive indices. The experimentally measured temperature-dependent real indices of E7 at frequencies of $0.34,0.70,0.98$, and $1.40 \mathrm{THz}$ and the fitting curves (solid lines) are plotted as functions of $T-T_{c}$ in Fig. 9. The filled and open circles are experimental data for $n_{e}$ and $n_{o}$, respectively. Above the transition temperature, i.e., in the isotropic phase, the experimental data are shown in Fig. 9 as crosses. Below the transition tempera-
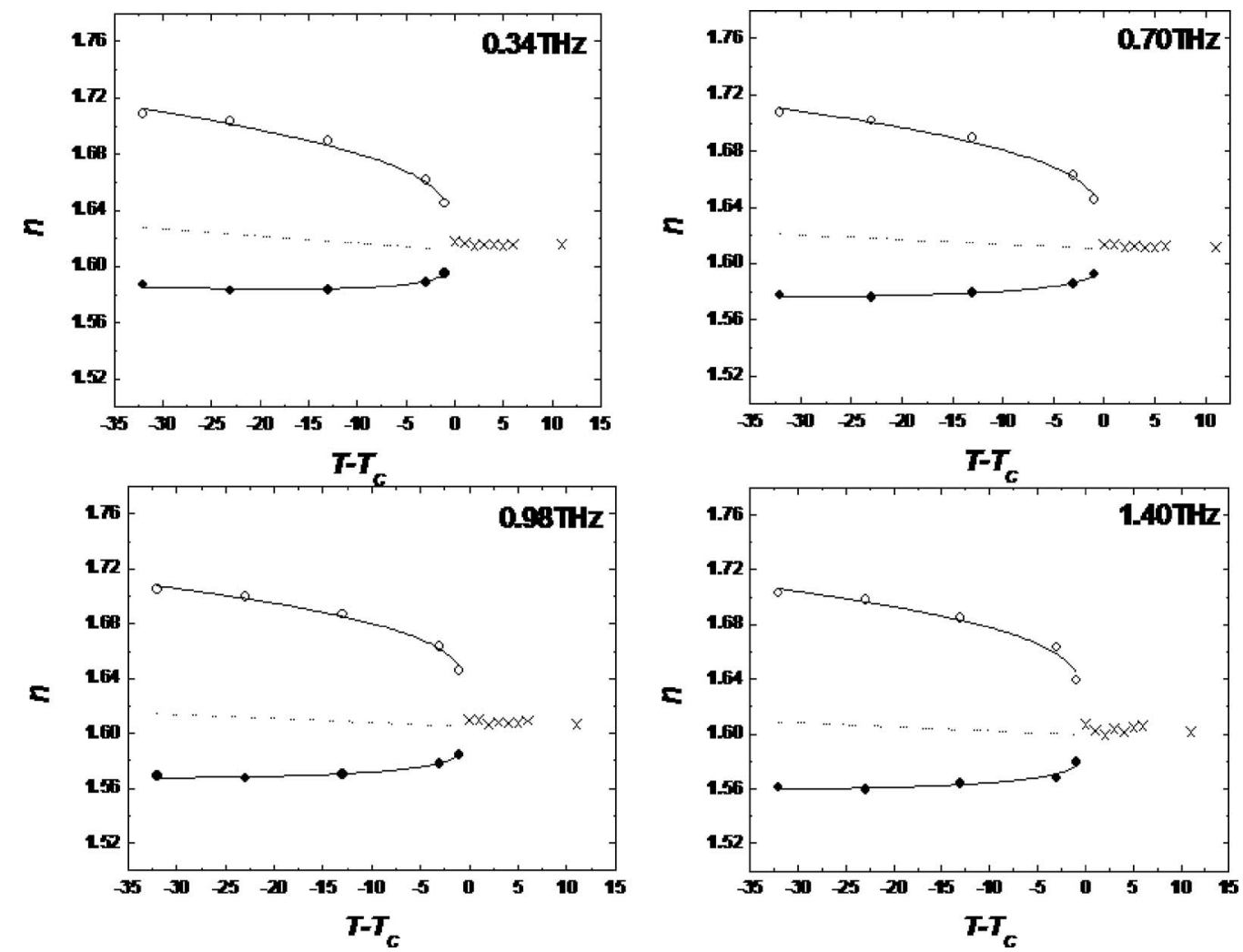

Fig. 9. Extraordinary and ordinary refractive indices of E7 versus $T-T_{c}$ at frequencies of $0.34,0.70,0.98$, and $1.40 \mathrm{THz}$. The open and closed circles represent $n_{e}$ and $n_{o}$, respectively. The crosses represent the refractive indices of E7 in the isotropic phase. The solid lines are the fitting curves. The dashed lines are the average indices at temperatures below $T_{c}$. 


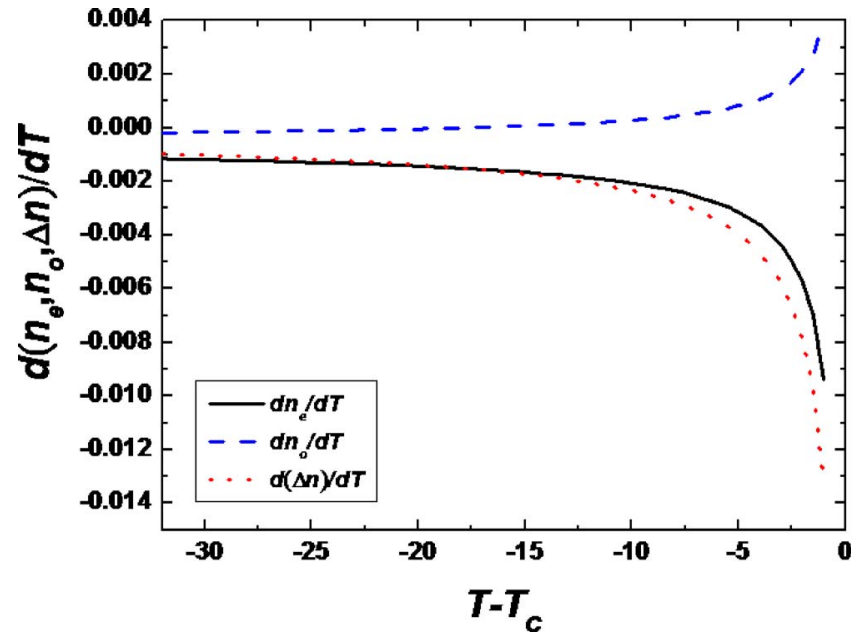

Fig. 10. (Color online) Temperature dependence of $\mathrm{d} n_{e} / \mathrm{d} T$, $\mathrm{d} n_{o} / \mathrm{d} T$, and $\mathrm{d}(\Delta n) / \mathrm{d} T$ of $\mathrm{E} 7$ at $0.34 \mathrm{THz}$. The solid and dashed curves represent $\mathrm{d} n_{e} / \mathrm{d} T$ and $\mathrm{d} n_{o} / \mathrm{d} T$, respectively. The dotted curve is for $\mathrm{d}(\Delta n) / \mathrm{d} T$.

ture, the dashed lines show the average indices, $\left(2 n_{o}\right.$ $\left.+n_{e}\right) / 3$, calculated from the fitting results.

The temperature gradients of $n_{e}, n_{o}$, and $\Delta n$ for E7 can be calculated by using Eqs. (12) and (13) and the parameters listed in Tables 1 and 2. These are shown in Fig. 10. Both $n_{e}$ and $\Delta n$ have negative temperature gradients, while that of $n_{o}$ exhibits a crossover behavior, i.e., it first decreases with temperature around room temperature and then increases with temperature. When the temperature approaches $T_{c}$, all of these three quantities change abruptly as shown in Fig. 10.

\section{CONCLUSIONS}

We have investigated the terahertz optical constants of E7, a liquid crystal (LC) mixture widely used across the electromagnetic spectrum for various applications. For the frequency range of $0.2-2.0 \mathrm{THz}$, the real parts of the extraordinary and ordinary indices of refraction of E7 at $26^{\circ} \mathrm{C}$ are $1.690-1.704$ and $1.557-1.581$, respectively. The birefringence of $\mathrm{E} 7$ is $0.130-0.148$ in this frequency range. The optical constants of $\mathrm{E} 7$ in the terahertz or submillimeter wave range are found to deviate significantly from values predicated by the usual extended Cauchy equations used in the visible and near-infrared. We also show that E7 is dichroic. The imaginary parts of the indices of refraction at room temperature are $\kappa_{e}$ $=0.010-0.020$ and $\kappa_{o}=0.028-0.042$. There is no sharp absorption feature for E7 from 0.2 to $2.0 \mathrm{THz}$. We have investigated the effect of temperature on the terahertz optical constants and $\Delta n$ of $\mathrm{E} 7$ in the nematic and isotropic phases $\left(26^{\circ} \mathrm{C}-70^{\circ} \mathrm{C}\right)$. The nematic-isotropic phase transition is clearly demonstrated. The temperaturedependent order parameter extracted from $\Delta n$ agrees with values from the visible, near-infrared, and intermediate infrared regions quite well. We found that the temperature gradient of $n_{e}$ for E7 is negative, while that of $n_{o}$ changes from negative at room temperature into a large positive number as $T$ approaches $T_{c}$.

\section{ACKNOWLEDGMENTS}

This work was supported in part by grants No. 95-2218E-007-119-MY3 and No. 2815-C-009-003-E from the National Science Council. C.-S. Yang would like to thank I-Chen Ho for assistance and helpful discussions.

\section{REFERENCES}

1. S.-T. Wu and D.-K. Yang, Fundamentals of Liquid Crystal Devices, Wiley Series in Display Technology (Wiley, 2006).

2. B. Bahadur, Liquid Crystals-Applications and Uses (World Scientific, 1992).

3. J. Li, C.-H. Wen, S. Gauza, R. Lu, and S.-T. Wu, "Refractive indices of liquid crystals for display applications," J. Disp. Technol. 1, 51-61 (2005).

4. J. Li, S.-T. Wu, S. Brugioni, R. Meucci, and S. Faetti, "Infrared refractive indices of liquid crystals," J. Appl. Phys. 97, 073501 (2005)

5. S.-T. Wu, "Birefringence dispersions of liquid crystal," Phys. Rev. A 33, 1270-1274 (1986).

6. S. Brugioni and R. Meucci, "Liquid crystals in the midinfrared region and their applications," Infrared Phys. Technol. 46, 17-21 (2004).

7. B. Ferguson and X.-C. Zhang, "Materials for terahertz science and technology," Nature Mater. 1, 26-33 (2002).

8. M. Tonouchi, "Cutting-edge terahertz technology," Nat. Photonics 1, 97-105 (2007).

9. Y. J. Ding, Q. Hu, M. Kock, and C. E. Stutz, eds., "Special issue on THz materials, devices, and applications," IEEE J. Sel. Top. Quantum Electron. 14, 257-259 (2008).

10. X.-C. Zhang, R. Beigang, and K. Tanaka, eds., "Special issue on THz wave photonics," J. Opt. Soc. Am. B 25, A1A125 (2009).

11. P. F. Goldsmith, "Quasi-optical techniques," Proc. IEEE 80, 1729-1747 (1992).

12. R. Kersting, G. Strasser, and K. Unterrainer, "Terahertz phase modulator," Electron. Lett. 36, 1156-1158 (2000).

13. H.-T. Chen, W. J. Padilla, M. J. Cich, A. K. Azad, R. D. Averitt, and A. J. Taylor, "A metamaterial solid-state terahertz phase modulator," Nat. Photonics 3, 148-151 (2009).

14. C.-Y. Chen, C.-F. Hsieh, Y.-F. Lin, R.-P. Pan, and C.-L. Pan, "Magnetically tunable room-temperature $2 \pi$ liquid crystal terahertz phase shifter," Opt. Express 12, 2625-2630 (2004).

15. C.-Y. Chen, C.-L. Pan, C.-F. Hsieh, Y.-F. Lin, and R.-P. Pan, "Liquid-crystal-based terahertz tunable Lyot filter," Appl. Phys. Lett. 88, 101107 (2006).

16. I.-C. Ho, C.-L. Pan, C.-F. Hsieh, and R.-P. Pan, "Liquidcrystal-based terahertz tunable Solc filter," Opt. Lett. 33, 1401-1403 (2008).

17. C.-L. Pan, C.-F. Hsieh, R.-P. Pan, M. Tanaka, F. Miyamaru, M. Tani, and M. Hangyo, "Control of enhanced THz transmission through metallic hole arrays using nematic liquid crystal," Opt. Express 13, 3921-3930 (2005).

18. S. A. Jewell, E. Hendry, T. H. Issac, and J. R. Sambles, "Tuneable Fabry-Perot etalon for terahertz radiation," New J. Phys. 10, 033012 (2008).

19. C.-F. Hsieh, Y.-C. Lai, R.-P. Pan, and C.-L. Pan, "Polarizing terahertz waves with nematic liquid crystals," Opt. Lett. 33, 1174-1176 (2008).

20. C.-J. Lin, Y.-T. Li, C.-F. Hsieh, R.-P. Pan, and C.-L. Pan, "Manipulating terahertz wave by a magnetically tunable liquid crystal phase grating," Opt. Express 16, 2995-3001 (2008).

21. R. Wilk, N. Vieweg, O. Kopschinski, and M. Koch, "Liquid crystal based electrically switchable Bragg structure for THz waves," Opt. Express 17, 7377-7382 (2009).

22. S.-T. Wu and K.-C. Lim, "Absorption and scattering measurements of nematic liquid crystals," Appl. Opt. 26, 17221727 (1987).

23. R.-P. Pan, C.-F. Hsieh, C.-L. Pan, and C.-Y. Chen, "Temperature-dependent optical constants and birefrin- 
gence of nematic liquid crystal 5CB in the terahertz frequency range," J. Appl. Phys. 103, 093523 (2008).

24. J. Li, S. Gauza, and S.-T. Wu, "Temperature effect on liquid crystal refractive indices," J. Appl. Phys. 96, 19-24 (2004).

25. J. Li, S. Gauzia, and S.-T. Wu, "High temperature-gradient refractive index liquid crystal," Opt. Express 12, 2002-2010 (2004).

26. N. Vieweg, C. Jansen, M. K. Shakfa, M. Scheller, N. Krumbholz, R. Wilk, M. Mikulics, and M. Koch, "Molecular properties of liquid crystals in the terahertz frequency range," Opt. Express 18, 6097-6107 (2010).

27. G. Abbate, V. Tkachenko, A. Marino, F. Vita, M. Giocondo, A. Mazzulla, and L. De Stefano, "Optical characterization of liquid crystals by combined ellipsometry and half-leakyguided-mode spectroscopy in the visible-near infrared range," J. Appl. Phys. 101, 073105 (2007).

28. F. Z. Yang and J. R. Sambles, "Determination of the micro- wave permittivities of nematic liquid crystals using a single-metallic slit technique," Appl. Phys. Lett. 81, 20472049 (2002)

29. F. Z. Yang and J. R. Sambles, "Determination of the permittivity of nematic liquid crystals in the microwave region," Liq. Cryst. 30, 599-602 (2003).

30. F. Z. Yang and J. R. Sambles, "Microwave liquid crystal wavelength selector," Appl. Phys. Lett. 79, 3717-3719 (2001).

31. T. S. Perova, "Far-infrared and low-frequency Raman spectra of condensed media," in Advances in Chemical Physics: Relaxation Phenomena in Condensed Matter, W. Coffey, eds. (Wiley, 1994), Vol. 87, pp. 427-480.

32. G. J. Evans and M. Evans, "High and low frequency torsional absorptions in nematic K21," J. Chem. Soc., Faraday Trans. 2 73, 285-292 (1977). 